\title{
BMJ Open CapeOX perioperative chemotherapy versus postoperative chemotherapy for locally advanced resectable colon cancer: protocol for a two-period randomised controlled phase III trial
}

\author{
Fangqi Liu, ${ }^{1,2}$ Tong Tong, ${ }^{2,3}$ Dan Huang, ${ }^{2,4}$ Weitang Yuan, ${ }^{5}$ Dechuan Li, ${ }^{6}$ \\ Jianjiang Lin, ${ }^{7}$ Sanjun Cai, ${ }^{1,2}$ Ye Xu, ${ }^{1,2}$ Wenbin Chen, ${ }^{7}$ Yueming Sun, ${ }^{8}$ Jing Zhuang ${ }^{9}$
}

To cite: Liu F, Tong T, Huang D, et al. Cape $0 \mathrm{X}$ perioperative chemotherapy versus postoperative chemotherapy for locally advanced resectable colon cancer: protocol for a twoperiod randomised controlled phase III trial. BMJ Open 2019;9:e017637. doi:10.1136/ bmjopen-2017-017637

- Prepublication history for this paper is available online. To view these files, please visit the journal online (http://dx.doi. org/10.1136/bmjopen-2017017637).

FL, TT and DH contributed equally.

Received 4 May 2017

Revised 20 October 2017 Accepted 24 October 2017

Check for updates

(C) Author(s) (or their employer(s)) 2019. Re-use permitted under CC BY-NC. No commercial re-use. See rights and permissions. Published by BMJ.

For numbered affiliations see end of article.

Correspondence to

Professor Sanjun Cai; caisanjuncsj@163.com and

Professor Ye Xu;

xuye021@163.com

\section{ABSTRACT}

Introduction Adjuvant chemotherapy with the Cape0X regimen is now widely used for treating colorectal cancer. However, prior studies have demonstrated better efficacy of pre-operative/neoadjuvant chemotherapy without increase of safety risks.

Methods and Analysis This multicentre, open-label, parallel-group, randomised, controlled, phase III study aims to compare the efficacy and safety of perioperative CapeOX chemotherapy with the postoperative one for treating patients with locally advanced $\mathrm{RO}$ resectable colon cancers in China. In total 1370 eligible patients will be randomised to: the test group, up to four cycles (every 3 weeks is a cycle, Q3W) of chemotherapy plus radical surgery plus up to four cycles of post-operative chemotherapy; or the control group, radical surgery first, then up to eight cycles of chemotherapy. In each cycle, oxaliplatin will be given at a dose of $130 \mathrm{mg} /$ $\mathrm{m}^{2}$ through continuous IV infusion for 2 hours on the first day. From day 1 to day 14 , capecitabine will be taken orally every morning and evening at a dose of $1000 \mathrm{mg} / \mathrm{m}^{2} / \mathrm{d}$. The primary outcome measure is the 3-year disease free survival. The objective response rate, $\mathrm{R} 0$ resection rate, overall survival, as well as the adverse events will also be measured as second endpoints. The study may include two periods. If results of period 1 are not favourable, period 2 will be initiated, recruiting genetically sensitive patients and repeating the same process with period 1 .

Ethics and dissemination Informed consent will be required from, and provided, by all subjects. The study protocol has been approved by the independent ethics committee of Shanghai Fudan University Cancer Centre. This study will clearly demonstrate the potential benefit of perioperative chemotherapy with the Cape $0 \mathrm{X}$ regimen. Results will be shared among all the participating centres, and with policymakers and the academic community to promote the clinical management of colon cancer. Trial registration number NCT03125980.

\section{INTRODUCTION}

Colorectal cancer is the third most common cancer in men $(663,000$ cases, $10.0 \%$ of the total) and the second in women $(570,000$ cases, $9.4 \%$ of the total) worldwide. ${ }^{1}$ Each

\section{Strengths and limitations of this study}

- An adequately powered, two-period, randomised and controlled trial;

- A second period to validate potentially sensitive patients based on data from the first period;

- A limited number of participating centres might limit extrapolations of study findings.

year, more than 1.2 million patients are diagnosed with colorectal cancer, and more than 600,000 die from the disease. ${ }^{2}$ In China, colorectal cancer ranks fifth among all kinds of cancers in terms of incidence and mortality, and the incidence has been increasing slightly year by year over the past decade. The gold standard for treating locally advanced colorectal cancer now is radical surgery plus postoperative 6-months FOLFOX/XELOX chemotherapy. This treatment, compared to surgery alone, has obviously improved disease-free survival and overall survival of colorectal cancer patients. A trial comparing the 10-year overall survival of patients with chemotherapy versus no chemotherapy after surgery has demonstrated the superiority of adjuvant therapy (46.9\% vs $29.9 \%$, $\mathrm{P}<0.001)$.

However, the postoperative therapy, to some extent, delays the systemic chemotherapy since it usually takes 3 months from diagnosis to starting chemotherapy. ${ }^{4-6}$ According to some professional opinions, one of the main reasons for metastasis after surgery is that the surgery stimulates and activates growth factors that cause rapid growth of the tumour. 6 The sooner the systemic chemotherapy is received, the better suppression it has on activity of growth factors, as well as eliminating tiny metastases. ${ }^{7-11}$ The neoadjuvant 
chemotherapy, provided before the radical surgery, may suppress tumour growth factors and decrease progression of micro-metastases. ${ }^{12}$ Another potential advantage of neoadjuvant chemotherapy is that it may shrink the invasion of the tumour, and thus reduce operational trauma and expedite recovery. ${ }^{13}$ This makes it more possible for patients to tolerate and receive large dosages for chemotherapy after the surgery. ${ }^{4}$ An increasing number of clinical trials have demonstrated even better efficacy of perioperative chemotherapy and radiotherapy than postoperative ones on locally advanced esophageal cancer, gastric cancer and rectal cancer. ${ }^{14-16}$ Feasibility and safety were also proved in some preliminary studies on the efficacy and safety of neoadjuvant chemotherapy for locally advanced colorectal cancer. ${ }^{12}$ 17-20

Unfortunately, the universal acceptance of neoadjuvant chemotherapy has been challenged by the clinical concerns that non-sensitive patients may have disease progression due to the delay of radical surgery and that incorrect computed tomography (CT) staging may lead to the overtreatment of low-risk patients. A crowd of clinical trials have showed no progression was developed in the process of the neoadjuvant chemotherapy. ${ }^{712} 19$ Moreover, the precision of pre-operative intestinal CT staging is proved in many imaging trials to be $41 \%$ to $82 \% .{ }^{21-25} \mathrm{As}$ shown in a trial conducted by Smith, ${ }^{25}$ pre-operative CT staging can provide accurate prognosis for non-metastatic colon cancer patients, and the accuracy is the same with that of histopathology. The researchers also suggested that pre-operative CT assessment of prognosis is helpful in identifying patients with poor prognosis and thus offering them neoadjuvant chemotherapy. On the other hand, continuous development of imaging, such as oral and enema contrast agent, makes pre-operative staging of colon cancer more precise, ${ }^{25}$ and so unnecessary chemotherapy can be avoided for low-risk patients.

The world is waiting for published results from phase III trials on neoadjuvant chemotherapy for locally advanced colorectal cancer. Very few phase III trials can be found registered on the international site of clinical researches. In China where there are a large number of patients with locally advanced colon cancer, not many data from largescale clinical trials have been published with respect to the neoadjuvant chemotherapy. The phase II trial conducted at Fudan University Shanghai Cancer Centre has yielded encouraging results $(\mathrm{N}=47$, CapeOX regimen, clinicaltrials.gov NCT02415829): after the neoadjuvant chemotherapy, no subject had disease progression and $68.1 \%$ subjects reached complete or partial response. Besides, the toxicity of neoadjuvant chemotherapy was acceptable. Based on those preliminary efficacy and safety data, we proposed to perform this prospective phase III randomised controlled trial with the expectation to find out a more effective treatment for patients with locally advanced colorectal cancer.

One of the factors that may impact the efficacy of therapy on end-stage colon cancer patients is the mutation of mismatch repair gene (MMR) (MLH1, MSH2,
MSH6, PMS2). Researchers have found that non-polyposis colon cancer demonstrated high rates of mutations, particularly in regions of short tandem repeats (named microsatellites), a phenomenon referred to as replication error or microsatellite instability (MSI). ${ }^{26-31}$. MSI has been shown to be correlated with better prognosis in patients with colorectal cancer. ${ }^{32}$ MSH6 protein expression in pretreatment biopsy tissue is an independent predictor for overall survival and MSH6 expression in resection specimens is an independent predictor for local recurrence in our analysis. Moreover, a trend for a better disease-free survival rate was observed in tumours with high expression of MSH2. ${ }^{33}$ In our study design, we will take into consideration the mismatch repair gene, and will screen out sensitive patients for a second period of trial if the first period fails to find superiority of neoadjuvant chemotherapy.

\section{METHODS AND ANALYSIS \\ Study design}

This phase III trial is to compare the efficacy and safety of perioperative adjuvant chemotherapy CapeOX versus postoperative chemotherapy CapeOX in patients who are diagnosed through CT with $\mathrm{T} 4$ or $\mathrm{N}+$ resectable colon cancer. It is hoped that the neoadjuvant systemic chemotherapy will lead to better clinical efficacy than the adjuvant chemotherapy by shrinking the tumour, lowering its staging, eliminating tiny metastases, and so on. The study will be conducted in about six study centres in China as a multicentre, randomised, open-label, parallel-group, active controlled superiority trial. This study adopts the Adaptive Signature Design, ${ }^{34}$ and is allowed to have two periods. The study phase scheme is shown in figure 1 , and the flow chart is summarised in figure 2.

After the first period, an interim analysis, under the supervision of the Independent Data Monitoring Committee (IDMC), will be performed to analyse the 3-year disease-free survival (3YDFS), as well as the overall survival, safety data, and so on. The IDMC will provide recommendations of go or not-go on period 2. If superiority of the neoadjuvant chemotherapy is identified and proved, the investigators will terminate the trial. Otherwise, new subjects will be recruited, and gene testing will be conducted to screen out sensitive patients for the second period of trial. Approximately 994 eligible subjects will be recruited in the first period, and approximately 376 subjects in the second period. Each of the two periods of the study may take up to 5 years, and consists of three consecutive parts for every subject: a 2-week screening and randomisation period; a 7 to 8 months' open-label treatment period; as well as relapse and survival follow-up of up to 4years.

The trial is planned to start in the middle of May 2017, and will necessitate a recruitment period of 2 to 3 years. After the screening, patients who are diagnosed with R0 resectable $\mathrm{T} 4 / \mathrm{N}+$ colon cancer and have provided written informed consent will be recruited for further eligibility 


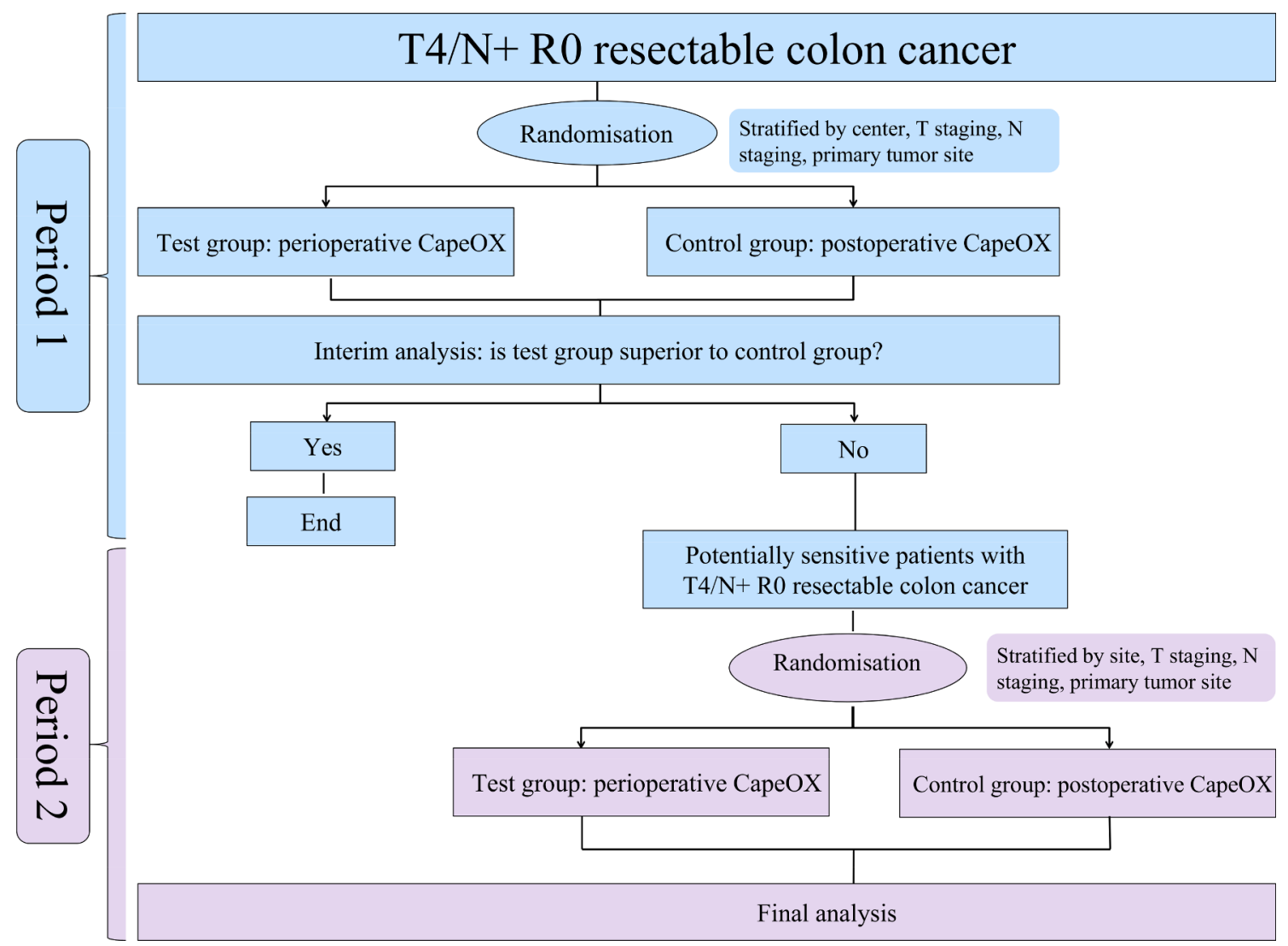

Figure 1. Phase scheme.

assessment. Eligible patients will then be allocated to the test group or the control group randomly at a 1:1 ratio and start receiving treatment immediately. Before the initiation of treatment, the biopsy procedure will be conducted for immunohistochemistry, and data of DNA MMR gene, MSI or RAS/BRAF will be collected. Biological specimens obtained in radical surgeries will also be used for immunohistochemistry test. Relevant data will be analysed to find out the potentially sensitive genes, based on which the patient screening in period 2 (if necessary) will be conducted.

Subjects in the test group will be given systemic CapeOX chemotherapy at first for up to four cycles (every 3 weeks is a cycle, Q3W), then have the radical surgery and rest for at least 4 weeks. Thereafter, they will receive postoperative CapeOX chemotherapy for at most four cycles (Q3W). Subjects in the control group will take the radical surgery first, and will then, after at least 4 weeks of rest, receive CapeOX chemotherapy for up to eight cycles (Q3W). In each cycle of chemotherapy, oxaliplatin will be given at a dose of $130 \mathrm{mg} / \mathrm{m}^{2}$ through continuous IV infusion for 2 hours on the first day. From day 1 to day 14, capecitabine will be taken orally every morning and evening at a dose of $1000 \mathrm{mg} / \mathrm{m}^{2} / \mathrm{d}$. The regimen is administered according to the NCCN guidelines for colon cancer. ${ }^{35}$ After the study treatment, long-term follow-up via outpatient visits and telephone visits will be performed for survival and tumour assessment.
The study data will be recorded by investigators in case reports (CRF), which will be reviewed and collected by independent data managers who do not participate in the clinical practice. CRFs will be stored by the sponsor/ research coordinator.

The study has been registered and the registration is ClinicalTrials.gov number, NCT03125980.

\section{Patients}

Patients should be diagnosed through CT scan with R0 resectable $\mathrm{T} 4 / \mathrm{N}+$ colon cancer. Specifically:

1. T staging:

T3: Rough intestinal wall(s) around the tumour.

T4a: Adjacent peritoneal nodular thickening.

T4b: Invasion of adjacent viscera.

2. $\mathrm{N}$ staging

a. Three lymph node aggregation.

b. The lymph node shows obvious enhancement, or inhomogeneous enhancement with rough edge.

c. Only one lymph node larger than $1 \mathrm{~cm}$.

3. Resectability: The lymph node resection is within the scope of D3 radical resection. The respectability of viscera for T4b patients will be determined through multi-disciplinary discussion.

Besides, they shall meet all the following inclusion criteria (except for the last one which is applicable only to period 2) for being included:

- Age: 18-75 years' old; 


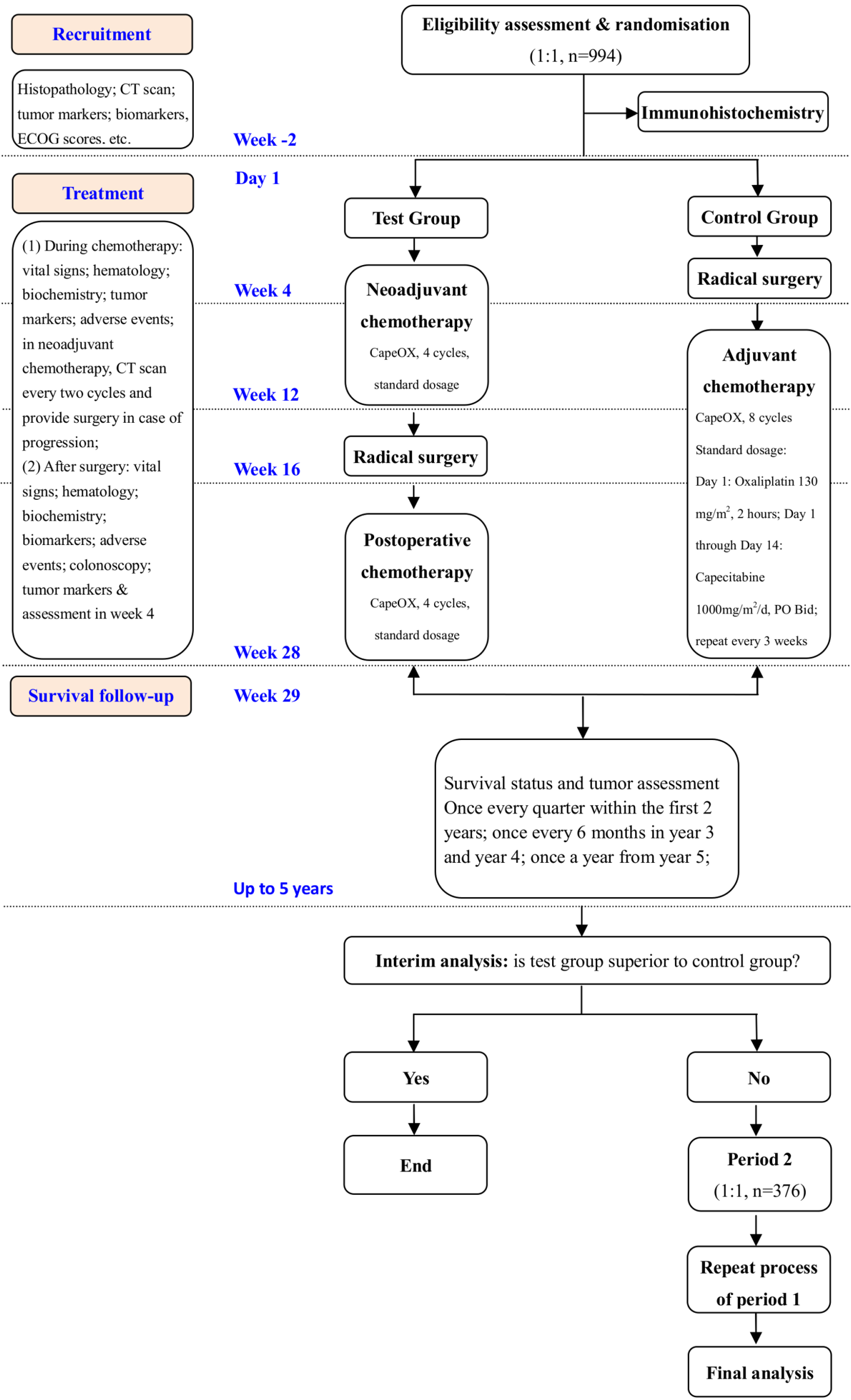

Figure 2. Flow chart. 
- Eastern Cooperative Oncology Group (ECOG) performance status (PS) $\leq 2$;

- Adequate bone marrow, hepatic and renal function as assessed by the following laboratory requirements conducted within 7 days of starting study treatment: neutrophil count $\geq 1.5 \times 109 / \mathrm{L}$; platelet count $\geq 100 \times 109 / \mathrm{L}$; haemoglobin $\geq 80 \mathrm{~g} / \mathrm{L}$; serum bilirubin $\leq 24 \mathrm{umol} / \mathrm{L}$; alanine aminotransferase and aspartate aminotransferase $\leq 60 \times \mathrm{IU} / \mathrm{L}$; ;serum creatinine $\leq 110 \mathrm{umol} / \mathrm{L}$;

- No current pregnancy or breast-feeding, and subjects of childbearing age shall take method of contraception;

- Be in a condition to receive a surgery/procedure;

- No second tumour at present or in the past 5 years, except skin basal cell carcinoma, skin squamous cell carcinoma or any in situ cancer;

- No previous systemic chemotherapy for treating colon cancer;

- No other chemotherapy at the same time;

- Expected lifetime longer than 3 months;

- Be willing and able to understand the study and to provide written informed consent.

- Other clinical factors (CEA) will also be considered for suspicious $\mathrm{T} 4 \mathrm{~b}$ patients, especially those with only one lymph node metastasis;

- Genetically sensitive to the chemotherapy (only for period 2);

Patients with any of the following conditions will be excluded from the study:

- End-stage cachexia patients;

- Cardiopulmonary dysfunction or liver and kidney dysfunction, and unable to tolerate CapeOX chemotherapy;

- Metastatic carcinoma;

- Moderate or above anaemia caused by serious local tumour bleeding;

- Incomplete or complete intestinal obstruction;

- Preoperative abscess formation;

- Known to be allergic to oxaliplatin or capecitabine;

- Active hepatitis, severe coagulation disorder patients;

- Pregnant or lactating women; or women who have fertility but have not taken adequate contraceptive measures;

- Known to have deficient dihydropyrimidine dehydrogenase (DPD);

- Have vital organ failure or other severe diseases, including, but not limited to, coronary heart disease, cardiovascular diseases or myocardial infarction within 12 months before being included; severe neurological or psychiatric history; severe infection; active disseminated intravascular coagulation;

- Unable or unwilling to abide by the study plan.

\section{Withdrawal management}

Subjects can decide to withdraw from the study at any time for whatever reason of their own. Visits and assessment will also be provided for withdrawal patients.
Investigators, on the other hand, can exclude patients who are affected by another disease, or cannot tolerate or suppress treatment-related toxicity, or have poor compliance or had better withdraw for the best medical profit.

Investigators are obliged to clarify reasons for withdrawal, and to confirm with subjects whether they would like to continue being contacted and providing survival data. Withdrawal reasons shall be recorded in case reports (CRF).

Unnecessary withdrawal will be avoided. Withdrawal patients are not allowed to participate in this trial any more.

\section{Premature termination}

The study may stop early under any of the following circumstances:

- Efficacy of the experiment group equals to, or worse than, the control group;

- Safety data are found to be unacceptable in the middle of the trial.

Investigators will assess all the subjects within at most 1 month after the decision is made for premature termination. All distributed but yet unused drugs and materials for the trial must be returned, and all the CRF must be completed as soon as possible.

\section{Recruitment process}

All study sites will participate in screening and recruiting patients, each of whom will be assigned a unique identification number. Patients screened out will be evaluated for eligibility according to inclusion and exclusion criteria by oncologists at the very site. All of the patients are outpatients.

\section{Randomisation process}

Randomisation will be conducted immediately after recruitment. Eligible subjects will be randomised into either the neoadjuvant group or the adjuvant group at a 1:1 ratio, and a second randomisation is not allowed for any patient. A stratified block randomisation will be adopted, and the sizes of blocks will vary randomly so as to avoid the predictability. To ensure a good balance between groups for distribution of important prognostic factors, stratification will be conducted as per:

- Study centre: one centre is a level;

- T staging: the level of T4 versus a level of non-T4;

- N staging: the level of N+ versus a level of non-N+;

- Primary tumour site: the level of left splenic flexure versus the level of right splenic flexure.

Random assignment is generated by an independent statistician from Shanghai Knowlands MedPharm Consulting Co., Ltd and assigned to each eligible patient via an interactive web-based response system. In order to avoid potential selection bias, the sequence is concealed from both clinical staff and patients until assignment.

All clinical practices after randomisation shall be recorded in CRF and shall be taken into consideration for analysis, no matter whether the randomised patients 
receive the study treatment or not. Study patients will not be treated without randomisation.

\section{Description of the intervention}

Test group

Patients in this group will be first given chemotherapy, then radical surgery and chemotherapy again after the surgery. Chemotherapy both before and after the surgery will use the CapeOX regimen at the same dosage for at most four cycles each, specifically:

- Oxaliplatin $130 \mathrm{mg} / \mathrm{m}^{2}$ iv continue for 2 hours.D1;

- Capecitabine $1000 \mathrm{mg} / \mathrm{m}^{2} / \mathrm{d}$ PO Bid, once every morning and evening. D1-14;

- Repeat every 3 weeks (Q3W).

After the surgery, patients have to take rest for at least 4 weeks before receiving chemotherapy. The chemotherapy is administered in accordance with the NCCN guideline for colon cancer. ${ }^{35}$ However, dosage can be modified and therapy can be delayed for at most 4 weeks for tolerance of, and recovery from, toxicity. During the pre-operative chemotherapy, a procedure will be provided in case of cancer progression, acute intestinal obstruction or perforation.

Medications used for both groups during study come from routine treatment, and require no extra package, labelling or storage.

\section{Control group}

Study patients in the control group will receive radical surgery first, and then rest for at least 4weeks. Thereafter, they will receive systemic chemotherapy with the CapeOX regimen for at most eight cycles. The chemotherapy is administrated in the same dosage with the test group.

The medication for study treatment will be recorded in CRF.

Adherence will be assessment at each study visit. Warnings will be given by investigators to subjects with poor adherence. Investigators are responsible for drug inventory management.

The surgical quality is secured for each group. The surgeons who will perform the surgical procedure at all the sites are highly experienced senior titles, that is chief physician or associate chief physician. Besides, quality control will be carried out through the pathological reports in terms of the number of lymph node dissection and circumferential resection margin, etc.

\section{STUDY VISITS}

To ensure adequate follow-ups and assessment, study visits are planned based on the study design and specific to the disease treatment:

- At baseline: diagnosis of colon cancer confirmed by histopathology examination: chest CT scan, abdomen CT scan and/or enhancement, abdominal/pelvic CT /MRI (scan+enhancement), tumour markers (carcinoembryonic antigen CEA, CA199, CA242, CA724,
CA50, alpha-fetoprotein AFP), biomarkers (MLH1, MSH2, MSH6, PMS2), ECOG score, and so on.

- During chemotherapy: haematology (white blood cells, neutrophils, platelet count, haemoglobin, and so on) once or twice every week; biochemistry (total bilirubin, alkaline phosphatase, AST, ALT, electrolyte [Na+, $\mathrm{K}+, \mathrm{CL}-, \mathrm{Mg} 2+, \mathrm{Ca} 2+])$, serum creatinine, creatinine clearance, urea, total protein, albumin) every 1 or 2 weeks; vital signs, haematology, biochemistry and tumour markers 3 days before each cycle; and CT scan every two cycles during neoadjuvant chemotherapy, and radical surgery will be performed in case of progression;

- After surgery: vital signs, wound healing, infection, inflammation, bleeding and complications on a daily basis; haematology and biochemistry on day 1, 3 and 5; biomarkers; and tumour markers and tumour assessment in week 4;

- After study treatment: check the following items every 3 months in the first 2 years, every 6 months from the third year through the fourth year, and every 12 months from the fifth year, until cancer regression or death: haematology and biochemistry, tumour markers, chest CT scan, abdominal/pelvic CT/MRI (scan + enhancement), tumour markers, and so on.

- Other post-operative follow-ups for primary colorectal cancer: electronic colonoscopy within 1 year after radical surgery; if abnormal, recheck within 1 year, otherwise, recheck every 5 years. More frequent colonoscopy check-up for subjects who are diagnosed with colon cancer at younger than 50 years. Colonoscopy check-up within 3 to 6 months after surgery for subjects who cannot take colonoscopy due to obstruction before the surgery.

All adverse effects happening or found during visits will be followed up until recovery, so will cancer-related symptoms until the first documented tumour progression.

No further anti-tumour therapy will be given until tumour progression.

\section{OUTCOME MEASURES}

All of the baseline, in-treatment and follow-up evaluations will be performed by experienced investigators during the study. The primary outcome measure is 3YDFS, defined as the length of time from the date of randomisation until the first documented date of relapse. The relapse will be judged by two experienced imaging experts based on results of such imaging tests as chest CT, abdominal-enhanced CT or enhanced MRI and PET scan in combination with physical examination and tumour marker examination. Puncture check or biopsy can be used if necessary.

In order to further assess the efficacy of the neoadjuvant chemotherapy, we will also measure the objective response rate by assessing the lesion shrinkage through CT scan before the surgery as per the RECIST version 1.1 - measurable lesions. ${ }^{36}$. After surgery, R0 resection 
rate (defined as the rate of patients whose tumours are completely resected with all the margins being negative) and TRG staging (defined as the TRG staging of tumour after surgery in line with AJCC) will be examined. Assessment of overall survival (defined as the length of time from randomisation date until the date of death from any cause) will also be performed.

Safety of the therapy will be evaluated through assessing adverse events according to the National Cancer Institute Common Terminology Criteria for Adverse Events (NCI CTCAE, VERSION 4.0). ${ }^{37}$ Any known serious adverse events must be reported by the investigator(s) to the sponsor/coordinator. A non-serious adverse event shall be reported within 5 working days, for which the report shall be signed and sent to the inspector or sponsor/coordinator. Analysis of adverse events (AEs) related to additional radiological imaging in the neoadjuvant group and genetic testing will be closely monitored.

\section{Sample size calculation}

Based on Curran et al's estimation, ${ }^{38}$ the sample size calculation for this study has taken into consideration the adjustment of testing significance level. Nominal significance levels are allocated at two-sided alpha levels of 0.04 and 0.01 for study period 1 and 2 respectively. ${ }^{34}{ }^{39}$ The primary outcome measure is 3YDFS.

\section{Sample size calculation for period 1}

Supposing that the 3YDFS of the study population is $60 \%$ with the adjuvant chemotherapy and will increase $9.2 \%$ (from $60 \%$ to $69.2 \%$ ) with the neoadjuvant chemotherapy, 318 events of death or progression will be required to observe, and the treatment comparison will be powered at $80 \%$ to establish differences for the primary endpoint, at a two-sided nominal significant level of $4.0 \%$ (corresponding to $\mathrm{HR}=0.72$ ). Sample size shall be $10 \%$ larger due to the possibility of drop-out. Therefore, a total of 994 subjects (497 subjects per group) will be enrolled and randomised to the test and the control group at a 1:1 ratio.

\section{Sample size calculation for period 2}

Assuming that the 3YDFS is $17.5 \%$ higher among sensitive subjects in the neoadjuvant chemotherapy group (test group), 106 events of death or regression will be observed. Differences can be identified with $80 \%$ power through the log-rank test at a two-sided nominal significant level of $1.0 \%$ (corresponding to $\mathrm{HR}=0.50$ ). A total of 376 potentially sensitive subjects ( 188 subjects per group) will be enrolled and randomised during this period.

\section{STATISTICAL ANALYSIS}

The primary outcome measure of this study is the 3YDFS, which will be analysed according to the intention to treat (ITT) principle through the two-sided log-rank test after the stratification factors are adjusted. The non-stratified log-rank test will also be conducted. Kaplan-Meier curves and estimates including median survival and confidence interval (CI) will be provided for each group. The 3-year and 5-year overall survivals will be analysed for both of the groups. Efficacy will be also demonstrated through hazard ratio with the use of the Cox proportional hazard model as appropriate. The plot of log-negative-log of the Kaplan-Meier estimates of the survival function versus the log of time and Schoenfeld residual versus time were used to visually check the proportional hazards assumption. ${ }^{40}$

Subgroup analysis will be performed if necessary with respect to efficacy targets. Subgroup stratification factors may include other important prognostic factors, such as organ function preservation for T4b patients and surgery quality (completeness of resection, quality of total mesorectal excision and edges after surgery), which will be determined in the statistical analysis plan (SAP). Hazard ratios and CIs of survival variables may be further presented with forest plots.

Safety will be analysed for all subjects receiving the study treatment. Summary statistics will be provided for AEs, which will be graded according to the criteria of NCI CTCAE V4.0. AEs of level 3 to 4 or with an incidence of $10 \%$ or higher will be listed and summarised, and between-group comparison of differences in AE incidences between the two groups will be performed through the two-sided Fisher test as appropriate. The Fisher test will also be considered for comparing study treatment interruption, delay and risk of death from toxicity due to AEs. Summary tables will be provided to describe continuous changes from baseline by treatment and visit. A shift table will also be provided for categorical laboratory parameters.

Logit model (machine learning will be adopted if applicable) will be set up to screen expressions of MMR (MLH1, MSH2, MSH6, PMS2), RAS and BRAF gene, based on which sensitivity to the neoadjuvant chemotherapy will be assessed. ${ }^{34}$ Parameters for screening out sensitive subjects will be calculated using relevant data obtained during period 1 by cross-validation.

The Logit model is constructed as below:

$$
\operatorname{Logit}\left(\mathrm{p}_{\mathrm{i}}\right)=\mu+\lambda_{\mathrm{j}} \mathrm{t}_{\mathrm{i}}+\beta_{\mathrm{j}} \mathrm{t}_{\mathrm{i}} \mathrm{x}_{\mathrm{i}, \mathrm{j}}
$$

Where, $\mathrm{p}_{\mathrm{i}}$ : probability of 3YDFS; $\lambda$ : main effect of treatment; $t$ : group $\left(\mathrm{t}_{\mathrm{i}}=1\right.$ represents the test group; $\mathrm{t}_{\mathrm{i}}=0$ represents the control group); $\beta$ : interaction effect of the specific group with the genetic expression; and $\mathrm{x}$ : the expression of the gene being assessed. If $\beta$ is statistically significant on specified test level $(\eta)$, the specific gene will be considered as being sensitive to treatment. The value of the specified test level is 0.1 .

Only those who meet both the sensitivity and inclusion criteria will be recruited and randomised in period 2.

$\mathrm{R}$, version 3.1.1 and SAS software, version 9.2 (SAS Institute, NC, USA) will be used for the statistical analysis. 


\section{Ethics and dissemination}

\section{Ethical considerations}

This study will be performed in accordance with the Declaration of Helsinki, without causing any extra harm or risks to patients. The protocol has been approved by the independent ethics committee of Fudan University Shanghai Cancer Centre for all the six centres (Approval No. 1703170-6). Written informed consents are required for patients to be enrolled.

\section{Relevance and dissemination}

The present phase III study aims at comparing the efficacy and safety of neoadjuvant CapeOX chemotherapy with the adjuvant chemotherapy for patients with locally advanced resectable colon cancer. There are only a handful of studies of its kind, without any published results available. Our adequately-powered randomised controlled trial, with two periods and more subjects ( $\mathrm{n}=994$ for period $1 ; \mathrm{n}=376$ for period 2), will be conducted in six centres with an expectation to demonstrate clear benefit of the perioperative chemotherapy with the CapeOX regimen.

First of all, the neoadjuvant chemotherapy is expected to be more effective than the adjuvant one for treating locally advanced colon cancer patients without raising risks or toxicity. The locally relapse and intraperitoneal implantation of colon cancer after surgery are closely related to the fallout and seeding of cancer cells during operation and the local resection degree. Chemotherapy before the surgery can shrink the tumour, and may reduce fallout of cancer cells ${ }^{41}$ and the rate of incomplete resection (Non-R0). ${ }^{42}$.Residual tumours from non-R0 resection are probably more aggressive and less sensitive to systemic chemotherapy. ${ }^{43}{ }^{44}$ There are no such risks with perioperative chemotherapy, and patients successfully receiving neoadjuvant chemotherapy are in a better condition for postoperative recovery ${ }^{13}$ and for larger dosages of adjuvant chemotherapy. ${ }^{4}$ Efficacy assessment of pre-operative chemotherapy may provide guidance for clinicians for optimised postoperative medication.

Second, data collected from this study can address the following concerns from clinical practices: whether the pre-operative CT staging is accurate enough to correctly identify different risks of patients in order to avoid overtreatment for low-risk patients; whether patients will develop progression or need emergency operation because of intestinal obstruction during pre-operative therapy; and whether the neoadjuvant chemotherapy is as safe as, or even safer than, the adjuvant one. Some trials have provided preliminary answers for those concerns. Hardly any patients develop progression during neoadjuvant chemotherapy, ${ }^{7} 1219$ and the accuracy of preoperative CT staging is $41 \%$ to $82 \% .{ }^{21-25}$ The single-arm randomised phase II study $(\mathrm{n}=47)$ conducted at Fudan University Shanghai Cancer Centre showed that no subjects had progression during preoperative chemotherapy with CapeOX, and $68.1 \%$ reached complete or partial response. ${ }^{45}$ However, more evidence and data are needed from larger-scale and/or phase III trials.

Moreover, this trial, adopting the Adaptive Signature Design ${ }^{34}$ will probably identify factors that impact the sensitivity of patients to CapeOX chemotherapy. Precision medical care has proved that only patients with certain mutations respond to a specific therapy. Mutations are considered in the second period of this trial, which will be conducted if the comparison in the first period fails to demonstrate a clear benefit of the neoadjuvant chemotherapy.

In summary, the neoadjuvant chemotherapy with the CapeOX regimen might be superior to the adjuvant one with respect to efficacy for patients with locally advanced colon cancer. The study findings will be shared with participating hospitals, policymakers and the academic community via conferences, data report and/or publication in order to promote the clinical management of locally advanced colon cancer in China and worldwide.

\section{Author affiliations}

${ }^{1}$ Department of Colorectal Surgery, Fudan University Shanghai Cancer Centre, Shanghai, China

${ }^{2}$ Department Of Oncology, Shanghai Medical College, Fudan University, Shanghai, China

${ }^{3}$ Department of Radiology, Fudan University Shanghai Cancer Centre, Shanghai, China

${ }^{4}$ Department of Pathology, Fudan University Shanghai Cancer Centre, Shanghai, China

${ }^{5}$ Department of Anorectal Surgery, The First Affiliated Hospital of Zhengzhou University, Zhengzhou, China

${ }^{6}$ Department of Colorectal Surgery, Zhejiang Cancer Hospital, Hangzhou, Zhejiang, China

${ }^{7}$ Department of Anorectal Surgery, The First Affiliated Hospital of Zhejiang University, Hangzhou, China

${ }^{8}$ Department of Colorectal Surgery, The First Affiliated Hospital of Nanjing Medical University, Nanjing, China

${ }^{9}$ Department of General Surgery, Zhengzhou University Cancer Hospital, Zhengzhou, China

Acknowledgements We would like to thank Sam Zhong (SZ) and Cuiling Yin (CLY) for their kind support with this protocol.

Contributors All the authors have contributed substantially to the draft and manuscript, and approved this submission. SJC, YX and FQL were responsible for the concept and design of the study. FQL, SJC and YX supervised the power and wrote the study design part. YX, TT and DH took overall responsibility for administration, ethical review and publication of the study. WTY, DCL, JJL, WBC, YMS and JZ are sub-centre principal investigators, and provided recruitment, technical and material support for the study.

Funding The authors have not declared a specific grant for this research from any funding agency in the public, commercial or not-for-profit sectors.

Competing interests None declared.

Patient consent for publication Not required.

Ethics approval The Ethics Committee of Shanghai Fudan University Cancer Centre.

Provenance and peer review Not commissioned; externally peer reviewed.

Open access This is an open access article distributed in accordance with the Creative Commons Attribution Non Commercial (CC BY-NC 4.0) license, which permits others to distribute, remix, adapt, build upon this work non-commercially, and license their derivative works on different terms, provided the original work is properly cited, appropriate credit is given, any changes made indicated, and the use is non-commercial. See: http://creativecommons.org/licenses/by-nc/4.0/. 


\section{REFERENCES}

1. Ferlay J, Shin HR, Bray F, et al. Estimates of worldwide burden of cancer in 2008: GLOBOCAN 2008. Int J Cancer 2010;127:2893-917.

2. Brenner $\mathrm{H}$, Kloor M, Pox CP. Colorectal cancer. Lancet 2014;383:1490-502.

3. Tsai WS, Hsieh PS, Yeh CY, et al. Long-term survival benefits of adjuvant chemotherapy by decreasing incidence of tumor recurrence without delaying relapse in stage III colorectal cancer. Int J Colorectal Dis 2011;26:1329-38.

4. Finlay IG, Meek D, Brunton F, et al. Growth rate of hepatic metastases in colorectal carcinoma. Br J Surg 1988;75:641-4.

5. Tanaka K, Shimada H, Miura M, et al. Metastatic tumor doubling time: most important prehepatectomy predictor of survival and nonrecurrence of hepatic colorectal cancer metastasis. World J Surg 2004;28:263-70.

6. Scheer MG, Stollman TH, Vogel WV, et al. Increased metabolic activity of indolent liver metastases after resection of a primary colorectal tumor. J Nucl Med 2008;49:887-91.

7. Zeamari S, Roos E, Stewart FA. Tumour seeding in peritoneal wound sites in relation to growth-factor expression in early granulation tissue. Eur J Cancer 2004;40:1431-40.

8. Fahmy RG, Dass CR, Sun LQ, et al. Transcription factor Egr-1 supports FGF-dependent angiogenesis during neovascularization and tumor growth. Nat Med 2003;9:1026-32.

9. van der Bij GJ, Oosterling SJ, Beelen $\mathrm{RH}$, et al. The perioperative period is an underutilized window of therapeutic opportunity in patients with colorectal cancer. Ann Surg 2009;249:727-34.

10. Dighe S, Purkayastha S, Swift I, et al. Diagnostic precision of CT in local staging of colon cancers: a meta-analysis. Clin Radiol 2010;65:708-19.

11. Dighe S, Swift I, Magill L, et al. Accuracy of radiological staging in identifying high-risk colon cancer patients suitable for neoadjuvant chemotherapy: a multicentre experience. Colorectal Dis 2012;14:438-44.

12. Arredondo J, Pastor C, Baixauli J, et al. Preliminary outcome of a treatment strategy based on perioperative chemotherapy and surgery in patients with locally advanced colon cancer. Colorectal Dis 2013;15:552-7.

13. Assersohn L, Norman A, Cunningham D, et al. Influence of metastatic site as an additional predictor for response and outcome in advanced colorectal carcinoma. Br J Cancer 1999;79:1800-5.

14. Sjoquist KM, Burmeister BH, Smithers BM, et al. Australasian gastrointestinal trials group. Survival after neoadjuvant chemotherapy or chemoradiotherapy for resectable oesophageal carcinoma: an updated meta-analysis. Lancet Oncol 2011;12:681-92.

15. Cunningham D, Allum WH, Stenning SP, et al. for the MAGIC Trial Participants. Perioperative chemotherapy versus surgery alone for resectable gastroesophageal cancer. N Engl J Med 2006;355:11-20.

16. Sebag-Montefiore D, Stephens RJ, Steele R, et al. Preoperative radiotherapy versus selective postoperative chemoradiotherapy in patients with rectal cancer (MRC CR07 and NCIC-CTG C016): a multicentre, randomised trial. Lancet 2009;373:811-20.

17. Foxtrot Collaborative Group. Feasibility of preoperative chemotherapy for locally advanced, operable colon cancer: the pilot phase of a randomised controlled trial. Lancet Oncol 2012;13:1152-60.

18. Jakobsen A, Andersen F, Fischer A, et al. Neoadjuvant chemotherapy in locally advanced colon cancer. A phase II trial. Acta Oncol 2015;54:1747-53.

19. Arredondo J, Baixauli J, Pastor C, et al. Mid-term oncologic outcome of a novel approach for locally advanced colon cancer with neoadjuvant chemotherapy and surgery. Clin Trans/ Oncol 2017;19:379-85.

20. Memorial Sloan-Kettering Cancer Center. Neoadjuvant FOLFOX plus bevacizumab chemotherapy in patients with locally advanced colon cancer. http://clinicaltrials.gov/ct2/show/NCT00826800

21. McAndrew MR, Saba AK. Efficacy of routine preoperative computed tomography scans in colon cancer. Am Surg 1999;65:205-8.
22. Barton JB, Langdale LA, Cummins JS, et al. The utility of routine preoperative computed tomography scanning in the management of veterans with colon cancer. Am J Surg 2002;183:499-503.

23. Gollub MJ, Schwartz LH, Akhurst T. Update on colorectal cancer imaging. Radiol Clin North Am 2007;45:85-118.

24. Chiesura-Corona M, Muzzio PC, Giust G, et al. Rectal cancer: CT local staging with histopathologic correlation. Abdom Imaging 2001;26:134-8

25. Smith NJ, Bees N, Barbachano Y, et al. Preoperative computed tomography staging of nonmetastatic colon cancer predicts outcome: implications for clinical trials. Br J Cancer 2007;96:1030-6.

26. Aaltonen LA, Peltomäki $P$, Leach FS, et al. Clues to the pathogenesis of familial colorectal cancer. Science 1993;260:812-6.

27. Ionov Y, Peinado MA, Malkhosyan S, et al. Ubiquitous somatic mutations in simple repeated sequences reveal a new mechanism for colonic carcinogenesis. Nature 1993;363:558-61.

28. Peltomäki P, Aaltonen LA, Sistonen P, et al. Genetic mapping of a locus predisposing to human colorectal cancer. Science 1993;260:810-2.

29. Thibodeau SN, Bren G, Schaid D. Microsatellite instability in cancer of the proximal colon. Science 1993;260:816-9.

30. Boland CR, Goel A. Microsatellite instability in colorectal cancer. Gastroenterology 2010;138:2073-87.

31. Lee V, Murphy A, Le DT, et al. Mismatch repair deficiency and response to immune checkpoint blockade. Oncologist 2016;21:1200-11.

32. Popat S, Hubner R, Houlston RS. Systematic review of microsatellite instability and colorectal cancer prognosis. J Clin Oncol 2005;23:609-18.

33. Huh JW, Kim HC, Kim SH, et al. Mismatch repair gene expression as a predictor of tumor responses in patients with rectal cancer treated with preoperative chemoradiation. Medicine 2016;95:e2582.

34. Freidlin B, Simon R. Adaptive signature design: an adaptive clinical trial design for generating and prospectively testing a gene expression signature for sensitive patients. Clin Cancer Res 2005;11:7872-8.

35. National Comprehensive Cancer Network. NCCN Clinical Practice Guidelines in Oncology Version 2.2014 Colon Cancer. https://www. nccn.org/professionals/physician_gls/f_guidelines.asp (accessed 15 Feb 2017).

36. Therasse P, Arbuck SG, Eisenhauer EA, et al. New guidelines to evaluate the response to treatment in solid tumors. European Organization for Research and Treatment of Cancer, National Cancer Institute of the United States, National Cancer Institute of Canada. J Natl Cancer Inst 2000;92:205-16.

37. National Cancer Institute (USA). Common Terminology Criteria for Adverse Events (CTCAE) version 4.0(2010). 2017. http://evs.nci.nih. gov/ftp1/CTCAE/About.html (accessed 2 Jan 2017).

38. Curran D, Sylvester RJ, Hoctin Boes G. Sample size estimation in phase III cancer clinical trials. Eur J Surg Oncol 1999;25:244-50.

39. Moyé LA. P-value interpretation and alpha allocation in clinical trials. Ann Epidemiol 1998;8:351-7.

40. Hosmer DW, Lemeshow S. Applied survival analysis: regression modeling of time to event data: John Wiley \& Sons, 1999.

41. Schelfhout VR, Coene ED, Delaey B, et al. The role of heregulin-alpha as a motility factor and amphiregulin as a growth factor in wound healing. J Pathol 2002;198:523-33.

42. Nelson H, Petrelli N, Carlin A, et al. Guidelines 2000 for colon and rectal cancer surgery. J Natl Cancer Inst 2001;93:583-96.

43. Quirke P, Durdey P, Dixon MF, et al. Local recurrence of rectal adenocarcinoma due to inadequate surgical resection. Histopathological study of lateral tumour spread and surgical excision. Lancet 1986;2:996-9.

44. Nomura K, Miyagawa S, Harada H, et al. Relationship between doubling time of liver metastases from colorectal carcinoma and residual primary cancer. Dig Surg 1998;15:21-4.

45. Liu F, Yang L, Wu Y, et al. CapeOX as neoadjuvant chemotherapy for locally advanced operable colon cancer patients: a prospective single-arm phase II trial. Chin J Cancer Res 2016;28:589-97. 